\title{
Actividad investigadora de los residentes de medicina interna de Galicia
}

Research activity of the residents of internal medicine of Galicia

\author{
Iria Î́iguez, David Rubal, María Matesanz, Pablo Ventura, Ramón Rabuñal, Rafael Monte \\ Servicio de Medicina Interna. Hospital Universitario Lucus Augusti. SERGAS. Lugo
}

\section{Resumen}

OBJETIVO: Conocer la actividad investigadora de los residentes de medicina interna (MI) de Galicia.

MÉTOD0: encuesta remitida a los residentes (MIR) de Medicina Interna de Galicia en Mayo de 2011. Las variables analizadas fueron: comunicaciones a congresos, publicaciones en revistas, capítulos de libros, Diploma de Estudios Avanzados (DEA), tesis doctoral y participación en proyectos de investigación financiados. Los resultados se clasificaron según el año de residencia. RESULTADOS: participaron 39 residentes (68\% de los MIR de MI de Galicia): 9 MIR de $1^{\circ}$ año, 14 MIR $2^{\circ}, 6$ MIR $3^{\circ}, 6$ MIR $4^{\circ}$ y 4 MIR $5^{\circ}$. Se registró una media de comunicaciones a congresos autonómicos por residente de 5,.85 (DS 5.22), 2,51 (3.64) a congresos nacionales y $0,74(1,9)$ a internacionales. El $41 \%$ y el $87,2 \%$ no había participado en ninguna comunicación a congresos nacionales e internacionales, respectivamente. Se registraron un total de 29 publicaciones, con una media inferior a 1 publicación por residente. El 71,8\% no había participado en ninguna publicación. El 15\% estaba realizando o habían obtenido el DEA. No se observaron diferencias en la actividad investigadora en relación al avance en los años de residencia. Tampoco se observaron diferencias en cuanto a si los residentes procedían o no de hospitales universitarios.

CONCLUSIÓN: la actividad investigadora de los residentes de medicina interna de Galicia es deficiente. Es preciso conseguir una mayor implicación por parte de los residentes, tutores y unidades de docencia de los distintos hospitales y acometer las medidas necesarias para cambiar esta situación, estimulando actividades de formación y promoción de la investigación en los hospitales de Galicia.

PALABRAS CLAVE: Medicina Interna, Formación Postgraduada, Investigación.

\section{Introducción}

La medicina interna (MI) se define como una especialidad médica troncal de ejercicio fundamentalmente hospitalario que ofrece a los pacientes adultos una atención integral de sus problemas de salud, utilizando un abordaje médico en la prevención, diagnóstico, indicación terapéutica y seguimiento de las enfermedades del adulto1. La actividad investigadora se considera un pilar fundamental de la profesión médica y, por ende, de la actividad del internista y debe ser fomentada desde los inicios de nuestra formación. En este sentido, el programa formativo de residentes de medicina interna (MIR), elaborado por la Comisión Nacional de la Especialidad, recoge que la formación integral del internista exige la adquisición de conocimientos para desarrollar tareas docentes e investigadoras². En nuestro

\begin{abstract}
AIM: To know the research activity of the residents of internal medicine (MI) of Galicia.

METHOD: a questionnaire was sent to the residents (MIR) of internal medicine of Galicia in May 2011. Variables analyzed were: congress presentations, scientific papers, book chapters, degree in advanced studies, doctoral thesis and participation in financed projects of investigation. Results were classified according to the year of residence training.

RESULTS: 39 MIR answered the questionnaire (68\% of the MIR of MI of Galicia): 9 first year MIR, $14 \mathrm{MIR} 2^{\circ}, 6 \mathrm{MIR} 3^{\circ}, 6 \mathrm{MIR} 4^{\circ}$ and $4 \mathrm{MIR} 5^{\circ}$. The mean of presentations to autonomic congresses per resident was 5.85 (DS 5.22), 2,51 (3.64) to national congresses and 0,74 (1,9) to internationals. $41 \%$ and $87,2 \%$ had not participated in any presentation neither in national nor in international congresses, respectively. 29 scientific papers were registered, with the mean under 1 paper per resident. 71,8\% did not have any paper. $15 \%$ were undertaking the degree in advanced studies. No differences were found regarding the research activity according to the years of residence training. No differences were found according to the belonging of the MIR to university hospitals.

CONCLUSION: The research activity of the residents of Galicia is deficient. It is necessary to achieve a better implication from residents, tutors and hospital teaching units and to implant the measures to change this situation. We must stimulate activities of research education and promotion of investigation in the hospitals of Galicia.
\end{abstract}

KEY WORDS: Internal Medicine, Postgraduate Training, Research.

ámbito, la Sociedad Gallega de Medicina Interna reconoce la investigación como parte integral de modelo de práctica clínica, entendiendo que el internista debe implicarse en la producción científica ${ }^{3}$.

Los internistas españoles participan activamente en la comunicación de su actividad investigadora ${ }^{4,5}$. Sin embargo, cuando se les pregunta por las principales carencias y necesidades de los servicios de medicina interna, un 14,4\% señalan a la actividad investigadora y científica ${ }^{1}$. Por otra parte, su actividad publicadora parece ser inferior a la de otros especialistas, al menos en Galicia, como se extrae de un estudio que analizó los méritos de investigación y académicos que presentaron los diferentes especialistas clínicos en la oferta de empleo público del SERGAS de $2006^{6}$. 
Con respecto a la formación MIR, no existen datos sobre la actividad investigadora de los residentes de $\mathrm{Ml}$, tampoco en nuestra comunidad autónoma, aunque se asume que su participación en tareas de investigación es baja ${ }^{7}$. El objetivo de este estudio fue conocer la actividad investigadora de los residentes de MI de Galicia, en cuanto a comunicaciones a congresos, publicaciones científicas, participación en proyectos de investigación y tesis doctorales.

\section{Método}

Se ha analizado la actividad investigadora de los residentes de MI de Galicia mediante la realización de una encuesta, voluntaria y confidencial, autoadministrada. Las encuestas fueron remitidas a los tutores de Ml de todos los hospitales gallegos con residentes de dicha especialidad en Mayo de 2011, quienes las distribuyeron entre sus residentes. Posteriormente los datos fueron reenviados a nuestro centro.

Las variables analizadas fueron: comunicaciones a congresos autonómicos, nacionales e internacionales, publicaciones en revistas indexadas y en revistas no indexadas (nacionales e internacionales), capítulos de libros, Diploma de Estudios Avanzados (DEA) en curso o realizado, tesis doctoral en curso o realizada y la participación en proyectos de investigación financiados.

El periodo analizado comprendió desde el inicio de la residencia hasta mayo de 2011. Los resultados se ordenaron por años de residencia para todas las variables consideradas, y fueron incluidos independientemente del número de orden que ocupasen los autores en la lista de firmantes de Ios trabajos. Posteriormente se incluyeron, en una tabla de Excel para su análisis mediante el programa SPSS.

\section{Resultados}

En el momento de realización del estudio había 57 residentes de medicina interna en Galicia, de los cuales participaron un 68\% (39 residentes: 9 residentes de primer año (MIR1), 14 MIR 2 ${ }^{\circ}, 6 \mathrm{MIR}^{\circ}, 6 \mathrm{MIR} 4^{\circ}$ y 4 MIR 50).
La participación fue de un 100\% (5 residentes) en el Hospital Lucus Augusti de Lugo (HULA), Hospital Arquitecto Marcide (HAM) de Ferrol (5), Hospital Meixoeiro (5) y POVISA de Vigo (4), un 91\% (11) en el Complexo Hospitalario Universitario de A Coruña (CHUAC), un 80\% (4) en el Complexo Hospitalario de Ourense (CHOU) y Complexo Hospitalario de Pontevedra (3) (CHOP) y un 22\% (2) en el Complexo Hospitalario Universitario de Santiago (CHUS). No se recibió ninguna respuesta del Hospital Xeral-Cíes de Vigo.

Se registraron un total de 227 comunicaciones a congresos autonómicos, 99 a nacionales y 31 a internacionales. Los datos obtenidos respecto a la actividad comunicadora en congresos mostraron una mayor participación en congresos autonómicos (media 5,.85 por residente; DS 5,22) que en los congresos nacionales $(2,51$; DS 3,64) e internacionales $(0,74 ;$ DS 1,9$)$ (tabla 1). Sin embargo, la moda (valor más frecuente) para el total de comunicaciones a congresos autonómicos fue 2 y para el total de comunicaciones a nacionales e internacionales fue 0 . Así, los residentes que no habían participado en ninguna comunicación a congresos fueron: 5 (12,8\%) en congresos autonómicos, 16 (41\%) en congresos nacionales y $34(87,2 \%)$ en congresos internacionales. En total, 11 residentes $(28,2 \%)$ habían participado en 3 comunicaciones a congresos o menos.

Se registraron un total de 29 publicaciones indexadas, con una media global inferior a 1 publicación por residente (tabla 2). Debido al escaso número de publicaciones registradas, no se diferenció entre publicaciones nacionales e internacionales según el año de residencia. En total, 28 residentes (71,8\%) no había participado en ninguna publicación en el momento de la realización de la encuesta. Respecto a la elaboración de capítulos de libros, se registraron 12 capítulos.

Al comparar el número de comunicaciones, publicaciones y capítulos de libro en función de que el residente estuviera cursando los primeros años de residencia $\left(1^{\circ}, 2^{\circ} 03^{\circ}\right)$ o los últimos $\left(4^{\circ}\right.$ y $\left.5^{\circ}\right)$, no se observaron diferencias significativas (tabla 3), si bien se observó una amplia variabilidad en los

Tabla 1.Comunicaciones de los residentes de medicina interna de Galicia distribuidas por año de residencia según el tipo de congreso al que fueron presentadas

\begin{tabular}{|c|c|c|c|}
\hline & Autonómico & Nacional & Internaciona \\
\hline Año de residencia ( $n^{0}$ de residentes) & media (DS) & media (DS) & media (DS) \\
\hline MIR 1 (39) & $1,9(3)$ & $0,6(1)$ & $0,1(0,3)$ \\
\hline MIR 2 (30) & $2,6(2,9)$ & $1(1,5)$ & $0,4(1,4)$ \\
\hline MIR 3 (16) & $2(2,3)$ & $1,4(1,6)$ & $0,3(0,8)$ \\
\hline MIR 4 (10) & $2,6(2,9)$ & $1,5(1,7)$ & $0,5(1,3)$ \\
\hline MIR 5 (4) & $4(4,9)$ & $2(2,3)$ & $1,4(1,5)$ \\
\hline Total (39) & $5,8(5,2)$ & $2,5(3,6)$ & $0,7(2,4)$ \\
\hline
\end{tabular}


Tabla 2. Publicaciones en revistas indexadas de residentes de Ml de Galicia según el año de residencia

\begin{tabular}{|l|c|}
\hline Año de residencia $\left(\mathrm{n}^{0}\right.$ de residentes) & Media (DS) \\
\hline MIR 1 (39) & $0,1(0,4)$ \\
\hline MIR 2 (30) & $0,2(0,5)$ \\
\hline MIR 3 (16) & $0,6(1)$ \\
\hline MIR 4 (10) & $0,3(0,7)$ \\
\hline MIR 5 (4) & $1,8(2,9)$ \\
\hline Total (39) & $0,7(1,9)$ \\
\hline
\end{tabular}

datos (ej. Un MIR de $5^{0}$ año tiene 10 publicaciones, la $3^{\mathrm{a}}$ parte del total).

Cuando comparamos la actividad investigadora de los residentes de hospitales universitarios (CHUAC, CHUS, Meixoeiro, POVISA, Xeral-Cíes) frente a los no universitarios (HULA, HAM, CHOU, CHOP) no se observaron diferencias de interés en el número de comunicaciones a congresos ni de publicaciones (tabla 4).

Respecto a la actividad investigadora ligada a la carrera universitaria, 6 residentes (15\%) estaban realizando o habían obtenido el DEA, sin diferencias en función del año de residencia y ninguno había completado la tesis doctoral. No hubo diferencias entre hospitales universitarios/no universitarios respecto al número de DEAs en curso o realizados. El 12\% había participado en proyectos de investigación financiados como investigador colaborador.

\section{Discusión}

La investigación biomédica es beneficiosa para la sociedad y para los MIR, ya que permite mejorar la asistencia clínica y respalda tanto la educación médica como la formación continuada., Por otra parte, facilita la adopción de nuevas técnicas procedentes de la investigación internacional y puede atraer a personal altamente cualificado al sistema sanitario ${ }^{8}$. La actividad investigadora de los médicos en general, y de los internistas en particular ha sido motivo de análisis en diversos estudios. Cuando se mide la aportación científica de la medicina interna gallega hospitalaria a congresos nacionales e internacionales de nuestra especialidad, se observa una creciente incorporación de los internistas hospitalarios gallegos a la investigación clínica comunicada en congresos nacionales e internacionales. Dicha producción ha alcanzado un elevado grado de continuidad sobre todo en los campos de investigación cardiovascular e infecciosas-SIDA ${ }^{5}$. Sin embargo, se ha señalado que la actividad investigadora de los internistas gallegos es significativamente menor que la del resto de especialidades médicas. Esto es debido a la suma de múltiples factores, como son su actividad predominantemente clínica, una mayor presencia en hospitales comarcales respecto a los de tercer nivel y en áreas clínicas de gran presión asistencial con menos tradición publicadora, y la existencia de una menor cantidad de revistas específicamente

Tabla 3. Comparación de la actividad investigadora de residentes de medicina interna de Galicia según estén en los años iniciales o finales de su residencia

\begin{tabular}{|l|c|c|c|}
\hline & MIR $1^{\circ}, 2^{\circ}, 3^{\circ}$ año $(\mathrm{n}=29)$ & MIR $4^{0}, 5^{\circ}$ año $(\mathrm{n}=10)$ & $\mathrm{p}$ \\
\hline Comunicación autonómica & $5,3(4,1)$ & $7,5(7,5)$ & 0,3 \\
\hline Comunicación nacional & $1,7(2)$ & $4,9(5,8)$ & 0,1 \\
\hline Comunicación internacional & $0,2(0,7)$ & $2,3(4,2)$ & 0,2 \\
\hline Total comunicaciones & $7,1(5,5)$ & $14,7(16,7)$ & 0,1 \\
\hline Publicaciones & $0,3(0,5)$ & $2,1(3,3)$ & 0,1 \\
\hline Capítulos de libro & $0,4(0,8)$ & $1,3(2,6)$ & 0,3 \\
\hline
\end{tabular}

Tabla 4. Comparación de la actividad investigadora de los residentes de medicina interna de Galicia según pertenezcan a un Hospital universitario o no universitario

\begin{tabular}{|l|c|c|c|}
\hline & $\begin{array}{c}\text { Hospital Universitario } \\
(\mathrm{n}=22) \text { Media (DS) }\end{array}$ & $\begin{array}{c}\text { Hospital no Universitario } \\
(\mathrm{n}=17) \text { Media (DS) }\end{array}$ & $p$ \\
\hline Comunicación autonómica & $5,1(4,9)$ & $6,8(5,5)$ & 0,2 \\
\hline Comunicación nacional & $2,4(3,3)$ & $2,6(4,1)$ & 0,8 \\
\hline Comunicación internacional & $0,2(0,6)$ & $1,4(3,4)$ & 0,1 \\
\hline Total comunicaciones & $7,6(8,2)$ & $10,9(11,9)$ & 0,3 \\
\hline Publicación revista & $0,5(1,3)$ & $1,1(2,4)$ & 0,3 \\
\hline Capítulo libro & $1(1,9)$ & $0,2(0,7)$ & 0,07 \\
\hline
\end{tabular}

Hospitales Universitarios: CHUAC, CHUS, CHUVI (Hospital Xeral-Cíes y Meixoeiro) Hospitales no Universitarios: HULA, HAM, CHOU, CHOP, POVISA 
dedicadas a la medicina interna respecto a otras especialidades, lo que supone una dificultad añadida para publicar ${ }^{6}$. Existe la idea entre la comunidad investigadora biomédica de una baja participación de los MIR en trabajos de investigación. Las causas de este déficit son diversas: falta de motivación de los integrantes de los servicios médicos acreditados y de los propios MIR, orientación de la formación MIR hacia las facetas asistenciales, sobrecarga de trabajo de los MIR y déficits estructurales de los servicios médi$\cos ^{7}$. En una encuesta reciente realizada entre los residentes de Ml de Galicia, el 73\% creía que la participación de su servicio en actividades de investigación era inadecuada y hasta un $87 \%$ consideraba que su servicio se preocupaba poco por implementar su formación en este ámbito. A pesar de que el $73 \%$ le daba mucha o bastante importancia a la actividad investigadora, el 78\% no había publicado ningún artículo científico ${ }^{9}$. Estas opiniones no difieren mucho de las obtenidas en otros estudios similares realizados en otras comunidades autónomas como Cataluña o el País Vasco ${ }^{10-12}$. Los resultados de nuestro estudio traducen estas opiniones en realidades, mostrando que la actividad investigadora de los residentes de medicina interna de Galicia es claramente deficiente. Su participación en congresos nacionales e internacionales es escasa o testimonial y su actividad publicadora es muy reducida. Tampoco se aprecia un aumento de la actividad en relación con el avance en los años de residencia, al contrario de lo que ocurre en Cataluña ${ }^{10}, y$ el número de residentes que han iniciado sus estudios de doctorado es escaso.

Se observó una mayor concentración de actividad investigadora orientada hacia los congresos autonómicos con respecto a los nacionales e internacionales. Es evidente que el factor proximidad y de comodidad juega un papel importante, aunque deben considerarse también las dificultades de financiación de los MIR para acudir a dichos eventos 9 . Por otra parte, se constata una brecha amplia entre el número de comunicaciones científicas a congresos y el de publicaciones. Existen varias posibilidades que pueden explicar este resultado: déficit de calidad científica en los trabajos comunicados, falta de continuidad en el esfuerzo investigador 0 complacencia con el servicio médico de origen en el envío de comunicaciones.

Al contrario de lo que cabría esperar no se observaron diferencias de importancia entre la actividad investigadora de los hospitales universitarios y los no universitarios. La escasa representación de residentes del CHUS y de Vigo en la muestra puede sesgar estos resultados. En todo caso, las diferencias no habrían sido probablemente muy signi- ficativas a favor de los hospitales universitarios y los datos coinciden con los resultados de una encuesta previa ${ }^{9}$.

El estudio presenta varias limitaciones. Por un parte, la ya comentada escasa participación de algunos hospitales (CHUS, Xeral-Cíes). Por otra, un posible sesgo de memoria, es decir, los participantes pueden haberse olvidado de algún trabajo que hayan realizado, o bien haberse equivocado en el año de residencia de realización, unido a que no se comprobaron los datos recibidos (encuesta anónima). En el sentido contrario, asumimos que hay un sesgo de comunicación, puesto que probablemente muchos residentes figuren como firmantes en Ios mismos trabajos; esta circunstancia seguramente empeoraría de forma significativa los resultados del estudio.

En resumen, la actividad investigadora de los residentes de medicina interna de Galicia es manifiestamente mejorable. Es preciso conseguir una mayor implicación por parte de los residentes, tutores y unidades de docencia de los distintos hospitales y acometer las medidas necesarias para cambiar esta situación, estimulando actividades de formación y promoción de la investigación. Sería deseable la acreditación de un mínimo de producción científica de calidad en los hospitales docentes y la creación de redes colaborativas entre los diversos servicios de medicina interna, y de estos con las universidades.

\section{Bibliografía}

1. Estudio Socioprofesional de la Medicina Interna en España: prospectiva 2010. Madrid: Sociedad Española de Medicina Interna (SEMI), 2005

2. Programa oficial de especialidad de medicina interna. BOE $n^{0} 33$, miércoles 7 de febrero de 2007; 5755-59

3. La Medicina Interna como modelo de práctica clínica. XXV aniversario. Sociedade Galega de Medicina Interna. Santiago de Compostela 2008

4. Montes-Santiago J, Formiga F. Investigación clínica española en insuficiencia cardiaca y su contribución a congresos europeos de medicina interna (1997-2009). Rev Clín Esp 2010; 210: 480-1

5. Montes Santiago J, Pérez Álvarez R. Aportación científica de la medicina interna hospitalaria a congresos nacionales e internacionales de su especialidad. An Med Interna (Madrid) 2002; 19: 567-70.

6. Rabuñal R, Monte R, Paz F, Bal M, López S, Arias C et al. Análisis de la actividad investigadora de los internistas gallegos a partir de los resultados de la oferta pública de empleo. An Med Interna (Madrid) 2007; 24: 365-8

7. Puerta JL, Moreno JM, Bravo S, Gutiérrez-Fuentes JA. Valoración de la investigación que se realiza en los hospitales españoles. Rev Clin Esp 2011; 211: 169-78.

8. Lewinson G. Beneficios de la investigación médica para la sociedad. Med Clin (Barc) 2008; 131 (suppl 5): 42-7

9. Meijide $H$, Rabuñal R, De la Iglesia F. La formación de residentes de medicina interna en Galicia: encuesta de opinión. Galicia Clin 2010; 71: 153-9.

10. Pujol R, Nogueras A. La formación de residentes de medicina interna. A propósito de una encuesta de opinión. Med Clin (Barc) 2002; 118: 545-50

11. Pijoan Jl, Urkaregi A, Moran J. Evaluación por los médicos internos residentes de la formación recibida en los servicios hospitalarios: una herramienta de monitorización. Gac Sanit 2001; 15: 432-40.

12. Giménez-Gómez N, Pedrazas D, Medina E, Dalmau D et al. Formación en investigación: autopercepción de los profesionales sobre sus necesidades. Med Clin (Bar) 2009; 132(3):112-117 